\title{
REPRESENTATION OF ABELIAN GROUPS AND RINGS BY FAMILIES OF REAL-VALUED FUNCTIONS
}

\author{
CARL W. KOHLS
}

\begin{abstract}
Sufficient conditions are indicated for the existence of an isomorphism of an abelian group or ring into the family of real-valued continuous functions on a realcompact or compact space. The spaces consist of sets of subsemigroups or subsemirings that are maximal among those containing a fixed element but not containing its additive inverse; in the ring case, where the element is the multiplicative identity, these are just infinite primes in the sense of Harrison. Earlier results of Harrison, Kadison, and Krivine follow from the present discussion.
\end{abstract}

Many representation theorems have been obtained for various algebraic systems-groups, vector spaces, rings, and algebras-stating that if a system satisfies certain conditions (usually involving a partial order), then it is isomorphic to a subsystem of the same kind in the family of real-valued continuous functions on a suitable Hausdorff space. For some examples, see [1], [5, Proposition 1.9], [6], and $[7$, p. 317]. Results for different systems are closely related; Kadison uses a representation of vector spaces to prove Stone's Theorem on algebras, while Dubois and Harrison apply the Kadison-Stone Theorem in obtaining their results on rings. These last proofs are striking in their use of an embedding of a ring into an algebra, but they rely on a norm and a completion. Our main purpose here is to show how a theorem that includes the results of Harrison and Krivine can be proved using a more algebraic approach resting ultimately on Hölder's Theorem about Archimedean totally ordered groups and on Zorn's Lemma. We first obtain a representation for abelian groups, and then apply it in the section on rings. A minor modification also yields Kadison's preliminary representation of partially ordered vector spaces. No new techniques are needed to prove our results.

The set of positive integers is denoted by $N$. If $S$ is a subset of an abelian group $G$, we write $-S$ for $\{g \in G:-g \in S\}$, and $|S|$ for $S \cap(-S)$. The notation $C(X)$ is used for the set of all real-valued

Received by the editors September 2, 1969.

A MS Subject Classifications. Primary 0678, 0680, 0685; Secondary 1640, 2030, 2080, 4625.

Key Words and Phrases. Ordered abelian group, ordered vector space, ordered ring, ordered algebra, Archimedean totally ordered group, representation, real-valued continuous function, realcompact space, compact space, Harrison infinite prime, extreme point. 
continuous functions on a topological space $X$; in $C(X)$, addition, multiplication, and order are defined pointwise. All topological spaces are taken to be completely regular Hausdorff spaces. A positive cone on a group or ring that induces a total order will be called a total cone. Most of the terminology introduced in the rest of the paper differs from that of other authors.

\section{Abelian groups.}

Definitions. 1. Let $e$ be an element in an abelian group $G$. An $e$-demigroup is a subsemigroup of $G$ that contains $e$ but does not contain $-e$.

2. An e-hemigroup is a maximal $e$-demigroup.

3. An $e$-demigroup $D$ is $N$-cancellative if $n a \in D, n \in N$ imply $a \in D$.

4. An e-demigroup $D$ is conic if $|D|=\{0\}$.

5. An $e$-demigroup $D$ is archimedean if $e+n a \in D$ for all $n \in N$ implies $a \in D$.

6. An $e$-demigroup $D$ is subarchimedean if $e+n a \in D$ for all $n$ in any infinite subset of $N$ implies $a \in D$.

7. Let $D$ be an $e$-demigroup. We say that $e$ is a (weak) $D$-unit if $a \in G(a \in D)$ implies $n e-a \in D$ for some $n \in N$.

Lemma 1. If $D$ is an e-demigroup containing 0 in an abelian group $G$, then $|D|$ is a subgroup, and $D /|D|$ is a conic $(e+|D|)$-demigroup in $G /|D|$.

The proof is routine.

Lemma 2. Let $H$ be an e-hemigroup in an abelian group $G$. Then:

(a) $H$ contains 0 ;

(b) $H /|H|$ is a conic $(e+|H|)$-hemigroup in $G /|H|$;

(c) $H$ is $N$-cancellative;

(d) $H \cup(-H)=G$;

(e) $H$ is archimedean.

Proof. (a) $H \cup\{0\}$ is an $e$-demigroup containing $H$, so $H=$ $H \cup\{0\}$.

(b) This follows easily from Lemma 1 and (a).

(c) First let $H$ be a conic $e$-hemigroup, and set $D=\{a \in G: n a \in H$ for some $n \in N\}$. Then $D$ is a semigroup containing $H$. If $-e \in D$, then for some $n \in N$, we have $n e \in|H|=\{0\}$, which implies $-e=(n-1) e$ $\in H$, a contradiction. Hence $D$ is an $e$-demigroup. Thus, $D=H$.

In general, if $n a \in H$ then $n(a+|H|) \in H /|H|$, so by (b) and what has just been proved, $a+|H| \in H /|H|$, whence $a \in H$.

(d) Suppose there is a $b \in G$ such that $b,-b \notin H$. Then $K=\{h+n b$ : $h \in H, n \in N \cup\{0\}\}$ is a semigroup containing $H$ and $b$, by (a). If 
$-e \in K$, then $-e=h+n b$ for some $h \in H, n \in N$, so $-e-n b \in H$, whence $-n b \in H$; this is impossible, by (c). Hence $K$ is an $e$-demigroup, a contradiction.

(e) Let $L=\{a \in G: e+n a \in H$ for all $n \in N\}$. Clearly $-e \notin L$. If $a, b \in L$, then $e+2 n a, e+2 n b \in H$ for all $n \in N$, so $2(e+n(a+b)) \in H$. By (c), $e+n(a+b) \in H$, whence $a+b \in L$. Hence $L$ is an $e$-demigroup containing $H$, which implies $L=H$.

LEMMA 3. Every subarchimedean e-demigroup is $N$-cancellative.

Proof. Let $D$ be a subarchimedean $e$-demigroup, and let $n a \in D$. Then $e+j n a \in D$ for all $j \in N$, so $a \in D$.

Remark 1. An archimedean $N$-cancellative $e$-demigroup $D$ is subarchimedean. For, assume that $e+n a \in D$ for all $n$ in some infinite subset $S$ of $N$, and let $j \in N$. Choosing $n \in S$ with $j<n$, we have $n e+n j a=(n-j) e+j(e+n a) \in D$, so $e+j a \in D$. Since $j$ is an arbitrary element of $N, a \in D$. Combining this result with Lemma 3 , we see that an archimedean $e$-demigroup is $N$-cancellative iff it is subarchimedean. In particular, every $e$-hemigroup is subarchimedean, by Lemma 2.

Lemma 4. Let $D$ be an $N$-cancellative e-demigroup in an abelian group $G$, and let $Y$ denote the set of e-hemigroups containing $D$. Then $\{a \in G: e+n a \in D$ for all $n \in N\}=\bigcap_{H \in Y} H$.

Proof. First assume that $e+n a \notin D$ for some $n \in N$. Then the semigroup generated by $D$ and $-(e+n a)$ does not contain $-e$; otherwise, we could write $-e=d-m(e+n a)$ for some $d \in D \cup\{0\}, m \in$ $N \cup\{0\}$, whence $m(e+n a) \in D$ and $m \neq 0$, so that $e+n a \in D$. Thus there is an $e$-hemigroup $H$ containing $D$ and $-(e+n a)$. Since $-e \notin H$, we have $n a \notin H$, so $a \in H$. Hence $a \notin \cap_{H \in Y} H$.

Now assume that $e+n a \in D$ for all $n \in N$, and let $H$ be an $e$-hemigroup containing $D$. Then by Lemma 2(e), $a \in H$. Thus $a \in \bigcap_{H \in Y} H$.

Lemma 5. Let $G$ be an abelian group with a conic e-demigroup $D$. Then there exists a unique isomorphism $\phi$ of $G$ into the additive group of real numbers with the natural order such that $\phi(D)$ is the total cone on $\phi(G)$ and $\phi(e)=1$ iff $D$ is an e-hemigroup such that $e$ is a weak $D$-unit.

Proof. First assume that $D$ is an $e$-hemigroup such that $e$ is a weak $D$-unit. By Lemma $2(\mathrm{~d}), D$ is a total cone on $G$. Let $a, b \in D$, with $b \neq 0$. Then $-b \in D$, so $-e=d-n b$ for some $d \in D, n \in N$, whence $n b-e \in D$. Also, there is a $j \in N$ such that $j e-a \in D$. Hence $j n b-a$ $=j(n b-e)+(j e-a) \in D$. Thus $G$ is an Archimedean totally ordered group, and the existence of $\phi$ follows from Hölder's Theorem [3, p. 
45]. Since the only order-preserving automorphism of a subgroup of the real numbers containing 1 that leaves 1 fixed is the identity, $\phi$ is unique.

The converse is easy to prove.

Theorem 1. Let $e$ be an element of an abelian group $G$, and let $X$ denote the set of all e-hemigroups $H$ in $G$ such that $e$ is a weak $H$-unit. Then there exist a realcompact topology on $X$ and a homomorphism $\psi$ of $G$ into the additive group $C(X)$ such that $D=\bigcap_{H \in X} H$ is the inverse image under $\psi$ of the positive cone on $\psi(G), \psi(e)=1$, and $\operatorname{Ker} \psi=\bigcap_{H \in X}|H|$.

If $e$ is a D-unit, then the topology on $X$ is compact.

Proof. The case in which $X$ is empty is trivial; so we assume it is nonempty. For each $H \in X, H /|H|$ is a conic $(e+|H|)$-hemigroup in $G /|H|$ and $e+|H|$ is a weak $H /|H|$-unit. By Lemma 5 , there is a unique homomorphism $\phi_{H}$ of $G$ into the additive group of real numbers such that $H$ is the inverse image under $\phi_{H}$ of the total cone on $\phi_{H}(G), \phi_{H}(e)=1$, and $\operatorname{Ker} \phi_{H}=|H|$. For each $c \in G$, let $R_{c}$ be a copy of the real line, and let $T$ be the subspace of the topological product $\prod_{c \in G} R_{c}$ consisting of all group homomorphisms $\eta$ of $G$ into the real numbers such that $\eta(e)=1$. It is easy to see that the inverse image of the nonnegative real numbers under any $\eta \in T$ is an element of $X$. Thus, $T=\left\{\phi_{H}: H \in X\right\}$. Now

$$
\begin{aligned}
T= & \bigcap_{(a, b) \in G \times G}\left\{f \in \prod_{c \in G} R_{c}: f(a+b)-f(a)-f(b)=0\right\} \\
& \cap\left\{f \in \prod_{c \in G} R_{c}: f(e)-1=0\right\} .
\end{aligned}
$$

For fixed $c \in G$, the projection $f \mapsto f(c)$ is continuous. Therefore $T$ is a closed subset of $\prod_{c \in G} R_{c}$. It follows that $T$ is realcompact $[4,11.12]$; we give $X$ the topology that makes the bijection $\phi_{I I} \mapsto H$ a homeomorphism.

For each $a \in G$, define a real-valued function $\psi(a)$ on $X$ by $(\psi(a))(H)$ $=\phi_{H}(a)$. Since $X$ has a topology that is induced by the product topology on $\prod_{c \in G} R_{c}$, it is immediate that $\psi(a) \in C(X)$. Clearly $\psi$ is a group homomorphism with the properties described in the statement of the theorem.

Now assume that $e$ is a $D$-unit. For any $H \in X$ and any $a \in G$, there is an $n_{a} \in N$ such that $n_{a} e \pm a \in D \subseteq H$. Hence $\phi_{H}\left(n_{a} e-a\right) \geqq 0$, $\phi_{H}\left(n_{a} e+a\right) \geqq 0$, and $-n_{a} \leqq \phi_{H}(a) \leqq n_{a}$. Since this is true for each $H \in X, T=\left\{\phi_{H}: H \in X\right\}$ is a closed subset of the compact space $\prod_{c \in G}\left[-n_{c}, n_{c}\right]$. It follows that the topology on $X$ is compact. 
Corollary 1. Let $D$ be a conic subarchimedean e-demigroup in an abelian group $G$, and let $X$ denote the set of all e-hemigroups $H$ in $G$ such that $e$ is a weak $H$-unit. Assume that each e-hemigroup containing $D$ is in $X$. Then there exist a realcompact topology on $X$ and an isomorphism $\psi$ of $G$ into the additive group $C(X)$ such that $\psi(D)$ is the positive cone on $\psi(G)$ and $\psi(e)=1$.

Proof. Let $Y$ denote the set of $e$-hemigroups containing $D$; by hypothesis, $Y \subseteq X$. By Lemma $3, D$ is $N$-cancellative, so by Lemma 4 , $\bigcap_{H \in Y} H=\{a \in G: e+n a \in D$ for all $n \in N\}=D$. Thus $\bigcap_{H \in Y}|H|=|D|$ $=\{0\}$, whence $\bigcap_{H \in X}|H|=\{0\}$.

Corollary 2. Let $D$ be a conic archimedean e-demigroup in an abelian group $G$ such that $e$ is a $D$-unit, and let $Y$ denote the set of all e-hemigroups containing $D$. Then there exist a compact topology on $Y$ and an isomorphism $\psi$ of $G$ into the additive group $C(Y)$ such that $\psi(D)$ is the positive cone on $\psi(G)$ and $\psi(e)=1$.

Proof. The proof of $[1$, Proposition 1$]$ shows that $D$ is $N$-cancellative. As in the proof of Corollary 1, this implies that $\bigcap_{H \in Y}|H|=\{0\}$. Let $X$ denote the set of all $e$-hemigroups $H$ in $G$ such that $e$ is a weak $H$-unit, so that $Y \subseteq X$. Then $\bigcap_{H \in X}|H|=\{0\}$, and Theorem 1 gives an isomorphism of $G$ into $C(X)$. Since $\bigcap_{H \in Y}|H|=\{0\}$ also, the homomorphism induced by restriction of functions in the image of $G$ to $Y$ is an injection, so the composition of these two mappings is the desired isomorphism $\psi$. Clearly $\psi(e)=1$; and since $D=\bigcap_{H \in Y} H, \psi(D)$ is the positive cone on $\psi(G)$.

Let $U=\left\{\phi_{H}: H \in Y\right\}, T=\left\{\phi_{H}: H \in X\right\}$, and $R_{c}$ be a copy of the real line for each $c \in G$. Then $U=\left(\bigcap_{a \in D}\left\{f \in \prod_{c \in G} R_{c}: f(a) \geqq 0\right\}\right) \cap T$, so $U$ is closed in $\prod_{c \in G} R_{c}$. As in the proof of Theorem 1,U is a subset of $\prod_{c \in a}\left[-n_{c}, n_{c}\right]$ for suitable $n_{c} \in N$, since $e$ is a $D$-unit. It follows that the topology on $Y$ is compact.

REMARK 2. If $G$ is a vector space over the real field, we define an $e$-demispace to be an $e$-demigroup that is closed under multiplication by nonnegative real numbers. The set $N$ can now be replaced by the set of positive real numbers, in particular in Definitions 5,6 , and 7 . Note that the concept of $N$-cancellative is superfluous here, and that subarchimedean is the same as archimedean. It is easy to see that our entire development can be carried over to this setting, simply making obvious modifications involving scalar multiplication, and noting in Lemma 5 that the order-preserving group isomorphism $\phi$ is now necessarily linear. In particular, a vector space with a conic archimedean $e$-demispace $D$ such that $e$ is a $D$-unit is the same as Kadison's 
archimedean partially ordered vector space with order unit $e$ (of positive dimension), and the analogue of Corollary 2 is [6, Lemma $2.5]$.

2. Rings. All rings are assumed to have an identity, denoted by 1 . For $n \in N, n 1$ is written simply as $n$.

Definitions. 8. A demiring in a ring $A$ is a subsemiring of $A$ that contains 1 but not -1 .

9. A hemiring is a maximal demiring.

Thus, a demiring is a 1-demigroup that is closed under multiplication. Note that if $D$ is a demiring in $A$ such that 1 is a $D$-unit, then $D-D=A$, so $|D|$ is an ideal in $A$.

The next result is essentially [5, Corollary 1.8$]$; we give an alternative proof.

TheOREM 2. Let $A$ be a ring with a conic demiring $P$. Then there exists a unique ring isomorphism $\psi$ of $A$ into the ring of real numbers with the natural order such that $\psi(P)$ is the total cone on $\psi(A)$ iff $P$ is a hemiring such that 1 is a P-unit.

Proof. First assume that $P$ is a hemiring such that 1 is a $P$-unit. Since $P$ is archimedean [5, Lemma A.5], by Corollary 2 there exist a compact topology on the set $Y$ of all 1-hemigroups containing $P$ and an additive group isomorphism $\psi$ of $A$ into the additive group $C(Y)$ such that $\psi(P)$ is the positive cone on $\psi(A)$ and $\psi(1)=1$.

We consider the space $U=\left\{\phi_{H}: H \in Y\right\}$ in the proof of Corollary 2 that is homeomorphic to $Y$. Then $U$ is a compact subset of the linear space $\prod_{c \in A} R_{c}$. Following [2, pp. 439-440], we say that a nonvoid subset $S$ of a subset $W$ of a linear space is an extremal subset of $W$ if $y, z \in W, 0<r<1$, and $r y+(1-r) z \in S$ imply $y, z \in S$. Note that $y$ is an extreme point of $W$ if and only if $\{y\}$ is an extremal subset of $W$. Let $E$ be the collection of all closed extremal subsets of $U$. It is easy to see that Zorn's Lemma can be applied to obtain a minimal element $M$ of $E$. Suppose there are two distinct points $\phi_{1}, \phi_{2} \in M$. Then there is some $c \in A$ such that the $c$ th coordinates of $\phi_{1}$ and $\phi_{2}$ are different. Let $k: T \rightarrow R_{c}$ be the projection mapping, and set $m=\min \{k(\phi): \phi \in M\}$, which exists since $M$ is compact. Let $M^{\prime}=\{\phi \in M: k(\phi)=m\}$. Then $M^{\prime}$ is a nonvoid closed proper subset of $M$, and it is easy to see that $M^{\prime}$ is an extremal subset of $M$, whence $M^{\prime} \in E$, a contradiction. Thus $U$ has an extreme point $\phi_{H}$.

Now let $a \in A$ be such that $\psi(a)$ is strictly positive and bounded by some positive integer $n$. Define $g_{1}, g_{2}$ by $g_{1}(b)=\phi_{H}(a b) / \phi_{H}(a), g_{2}(b)$ $=\phi_{H}((n-a) b) / \phi_{I I}(n-a)$ for all $b \in A$. Then $g_{1}, g_{2} \in U$ and $\phi_{H}(a) g_{1}(b)$ 
$+\phi_{H}(n-a) g_{2}(b)=\phi_{H}(a b)+\phi_{H}((n-a) b)=n \phi_{H}(b)$, so $g_{1}=\phi_{H}$, that is, $\phi_{H}(a b)=\phi_{H}(a) \phi_{H}(b)$ for all $b \in A$. Since each element of $A$ is the difference of elements that map under $\psi$ to strictly positive bounded functions, $\phi_{H}$ is multiplicative. But then $H$ is a demiring containing $P$, whence $P=H$, so that $X=\{H\}$ and $\psi$ is essentially the same as $\phi_{H}$.

Uniqueness follows as in Lemma 5, and the converse is again easy to prove.

Our final theorem includes the difficult half of [5, Proposition 1.9] and generalizes [7, p. 317].

Theorem 3. Let $A$ be a ring, and let $X$ denote the set of all hemirings $H$ in $A$ such that 1 is an $H$-unit. Then there exist a realcompact topology on $X$ and a homomorphism $\psi$ of $A$ into the ring $C(X)$ such that $D$ $=\bigcap_{H \in X} H$ is the inverse image under $\psi$ of the positive cone on $\psi(A)$ and $\operatorname{Ker} \psi=\bigcap_{H \in X}|H|$.

If 1 is a $D$-unit, then the topology on $X$ is compact.

The proof is similar to that of Theorem 1, with an appeal to Theorem 2 instead of to Lemma 5.

\section{REFERENCES}

1. D. W. Dubois, A note on David Harrison's theory of preprimes, Pacific J. Math. 21 (1967), 15-19. MR $35 \# 103$.

2. N. Dunford and J. T. Schwartz, Linear operators. I: General theory, Pure and Appl. Math., vol. 7, Interscience, New York, 1958. MR 22 \#8302.

3. L. Fuchs, Partially ordered algebraic systems, Addison-Wesley, Reading, Mass., 1963. MR $30 \# 2090$.

4. L. Gillman and M. Jerison, Rings of continuous functions, The University Series in Higher Math., Van Nostrand, Princeton, N. J., 1960. MR 22 \#6994.

5. D. K. Harrison, Finite and infinite primes for rings and fields, Mem. Amer. Math. Soc. No. 68 (1966). MR 34 \#7550.

6. R. V. Kadison, A representation theory for commutative topological algebra, Mem. Amer. Math. Soc. No. 7 (1951). MR 13, 360.

7. J. L. Krivine, Anneaux préordonnés, J. Analyse Math. 12 (1964), 307-326. MR 31 \#213.

Syracuse University, Syracuse, New York 13210 